\title{
When Less Is More: Non-monotonic Spike Sequence Processing in Neurons
}

\author{
Hinrich Arnoldt ${ }^{1,2}$, Shuwen Chang ${ }^{3}$, Sven Jahnke ${ }^{1,2,4}$, Birk Urmersbach ${ }^{1}$, \\ Holger Taschenberger ${ }^{3}$, Marc Timme $^{1,2,4 *}$
}

1 Network Dynamics, Max Planck Institute for Dynamics and Self-Organization (MPIDS), Göttingen, Germany, 2 Institute for Nonlinear Dynamics, Faculty of Physics, Georg August University Göttingen, Göttingen, Germany, 3 Department of Membrane Biophysics, Max Planck Institute for Biophysical Chemistry, Göttingen, Germany, 4 Bernstein Center for Computational Neuroscience (BCCN) Göttingen, Göttingen, Germany

* timme@nld.ds.mpg.de

\section{G openaccess}

Citation: Arnoldt $\mathrm{H}$, Chang S, Jahnke S, Urmersbach B, Taschenberger H, Timme M (2015) When Less Is More: Non-monotonic Spike Sequence Processing in Neurons. PLoS Comput Biol 11(2): e1004002. doi:10.1371/journal.pcbi.1004002

Editor: Lyle J. Graham, Université Paris Descartes, Centre National de la Recherche Scientifique, FRANCE

\section{Received: October 15, 2012}

Accepted: October 27, 2014

Published: February 3, 2015

Copyright: @ 2015 Arnoldt et al. This is an open access article distributed under the terms of the Creative Commons Attribution License, which permits unrestricted use, distribution, and reproduction in any medium, provided the original author and source are credited.

Funding: Partially supported by the BMBF Germany under grant no. 01GQ1005B and by a grant from the Max Planck Society to MT. The funders had no role in study design, data collection and analysis, decision to publish, or preparation of the manuscript.

Competing Interests: The authors have declared that no competing interests exist.

\section{Abstract}

Fundamental response properties of neurons centrally underly the computational capabilities of both individual nerve cells and neural networks. Most studies on neuronal input-output relations have focused on continuous-time inputs such as constant or noisy sinusoidal currents. Yet, most neurons communicate via exchanging action potentials (spikes) at discrete times. Here, we systematically analyze the stationary spiking response to regular spiking inputs and reveal that it is generically non-monotonic. Our theoretical analysis shows that the underlying mechanism relies solely on a combination of the discrete nature of the communication by spikes, the capability of locking output to input spikes and limited resources required for spike processing. Numerical simulations of mathematically idealized and biophysically detailed models, as well as neurophysiological experiments confirm and illustrate our theoretical predictions.

\section{Author Summary}

Brain function relies on robust communication between a huge number of nerve cells (neurons) that exchange short-lasting electrical pulses (called action potentials or spikes) at certain times. How nerve cells process their spiking inputs to generate spiking outputs thus is key not only to individual neurons' computational capabilities but also to the collective dynamics of neuronal networks. Here we analyze the response properties of neurons to regular spike sequence inputs. We find that neurons typically respond in a non-monotonic way. Output frequency mostly increases with input frequency as expected but sometimes output frequency necessarily decreases upon increasing the input frequency. Our theoretical analysis predicts that spiking neurons commonly exhibit such non-monotonic response properties. Simulations of simple mathematical and complex computational models as well as neurophysiological experiments confirm our theoretical predictions. 


\section{Introduction}

Most neurons in the nervous system communicate by sending and receiving stereotyped electrical pulses called action potentials or spikes [1]. The computational capabilities of neural circuits centrally rely on the input-output relations of single neurons. This relation is commonly characterized by its output spike rate in response to a temporally continuous constant input current $I$, sometimes in the presence of additional current fluctuations [2-5].

If the spikes each neuron receives are irregular in time and individually only weakly affect the neuron's membrane potential (the main physical quantity characterizing its dynamical state), this continuous-input picture serves as an appropriate approximation [6-8] to the actual spike sequence input. A neuron's response curve in terms of its output spike rate as a function of input current is thus considered one of its most fundamental standard characteristics [5, 9-11]. In particular, neurons are dynamically classified according to such response curves (so-called $f$-I-curves), into type-I neurons, with their output spike rates increasing from zero above a critical current $I_{c}$, and type-II neurons which start spiking with a macroscopic, nonzero rate upon increasing $I$ beyond some $I_{c}[10,11]$. For both types, neuronal output spike frequencies depend monotonically on the input $I$.

Yet, a broad range of neural systems, including most central pattern generators, pacemaker cells and neural pathways in the auditory and motor systems, exhibit more regular, patterned spike sequences [12-28] such that the above mean field approximation does not apply. Some experimental and numerical studies $[29,30]$ hint that certain neurons receiving regular periodic spiking inputs may exhibit locked spike responses together with possibly non-monotonic input-output relations. How common this phenomenon is and which neural features may cause non-monotonic response curves, however, remains unknown.

Here, joining theoretical, computational and experimental approaches we provide a systematic analysis of the response of individual neurons to regular spiking inputs. We find that nonmonotonic response is a universal feature that emerges due to a locking phenomenon [31] in a broad range of systems. We reveal the underlying mechanisms and conclude that an arbitrary system (neuronal or otherwise pulse-coupled) will inevitably show such non-monotonic response, if it exhibits pulsed outputs locked to the pulsed inputs and any form of resource limitation during pulse processing.

The article is structured as follows: we first derive analytical results on idealized neuron models; based on insights from this model, we identify general theoretical (sufficient) conditions underlying non-monotonicity. Second, we observe that these conditions commonly occur across neural systems and check their robustness against temporal jitter and their occurrence in biophysically more detailed models. Third, neurophysiological experiments confirm and illustrate our finding for real neurons.

\section{Results}

\section{Non-monotonic spike sequence responses abound}

Studying first the response properties of leaky integrate-and-fire (LIF) neurons [1] receiving periodic spiking input sequences via depressive synapses [32] (cf. Fig. 1), we find that slowly increasing the input frequency initially also increases the output spike frequency (as expected). When crossing certain input frequencies, however, the output frequency drops again, implying a non-monotonic response. Our extensive numerical studies show that this phenomenon robustly emerges for various combinations of neural time scales, synaptic time scales, synaptic efficacies and other features of the system. Checking various other types of neuron models including Fitzhugh-Nagumo and Hodgkin-Huxley neurons, this 


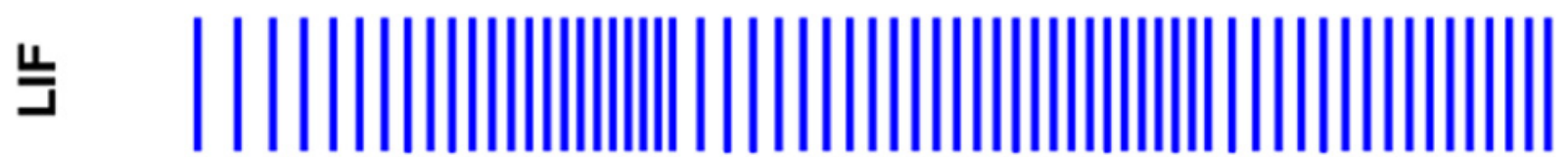

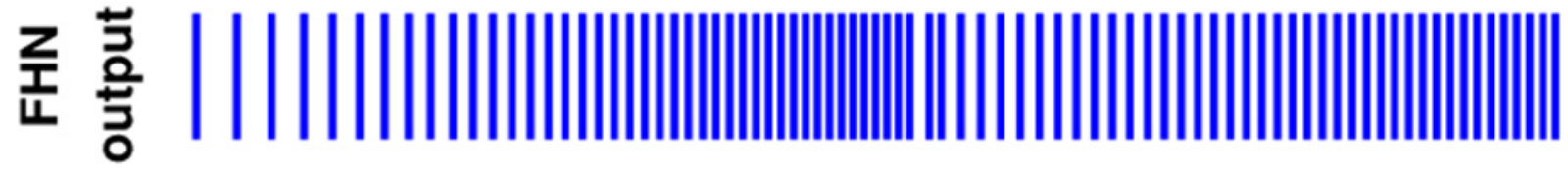

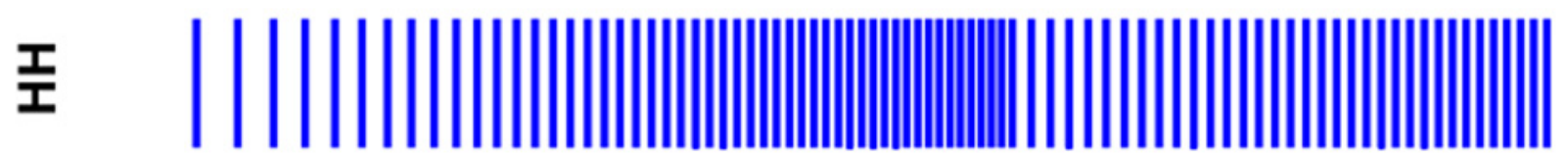

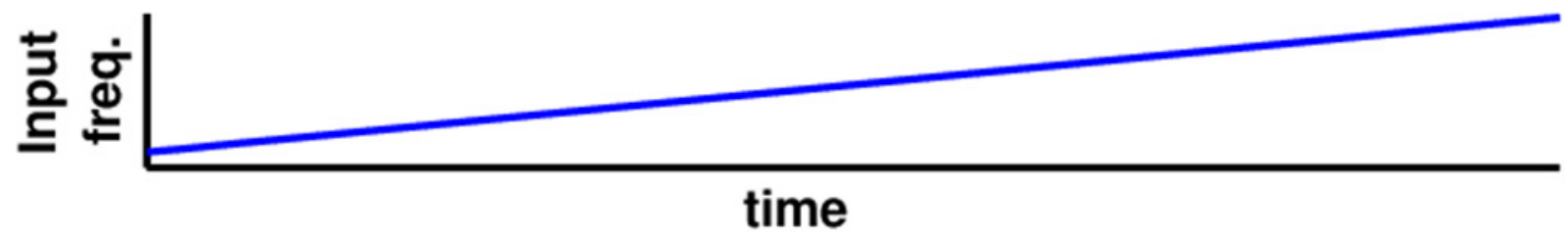

Figure 1. Non-monotonic response to regular input spike sequences: increasing the input spike frequency may increase but also decrease the output spike frequency. Bottom panel: input spike frequency that slowly increases ten-fold. Top three panels: output spike responses (LIF: leaky integrateand-fire neuron with depressive synapse, FHN: Fitzhugh-Nagumo and HH: Hodgkin-Huxley neuron, both with static synapses). Time is rescaled so that all three data sets fit in this Figure. For details of models see equations (1)-(2) for LIF, equations (12)-(13) for FHN and equations (14)-(17) for HH.

doi:10.1371/journal.pcbi.1004002.g001

phenomenon persists, even with static synapses. Fig. 1 illustrates three examples. These findings led us to hypothesize that non-monotonic responses to regular spike sequence inputs are a generic feature across neurons. To test this hypothesis, we first answered the question what causes such non-monotonic response.

\section{Theoretical analysis}

To reveal the basic ingredients underlying non-monotonic response, we analyzed an idealized, mathematically tractable system consisting of a LIF neuron receiving spiking input via a depressive synapse, i.e. a synapse that is weakened on short time scales when transmitting a pulse. The LIF-model [1] is a standard model for analyzing the dynamics of spiking neural systems and a standard model for depressive synapses was introduced by Tsodyks and Markram [32]. In their simplest setting, the combined dynamics of voltage $V(t)$ and synaptic resources $x(t)$ are given by

$$
\begin{aligned}
\dot{V} & =\frac{V_{\mathrm{eq}}-V}{\tau}+c x \sum_{m=-\infty}^{\infty} \delta\left(t-t_{m}\right), \\
\dot{x} & =\frac{1-x}{\mu}-u x \sum_{m=-\infty}^{\infty} \delta\left(t-t_{m}\right) .
\end{aligned}
$$

Here $\tau$ is the membrane time constant, $V_{\text {eq }}$ defines the equilibrium potential, the constant $c \in \mathbb{R}$ is the maximum possible response of the neuron to one incoming spike, it is modulated by the amount $x(t)$ of resources available at time $t$; further, $\mu$ is the time constant of resource recovery, 
$u$ is the fraction of available resources depleted per spike and $t_{m}$ defines the time of the $m$ th spike received by the neuron. Upon crossing a threshold $V\left(t^{-}\right) \geq V_{\Theta}:=1$, the neuron emits a spike and the membrane potential is reset, $V(t):=V_{\text {reset }}:=0$. With $V_{\text {eq }}<1$ the neuron is an excitable system and may only send a spike at times it receives an input spike.

What is the stationary spike response of this system to regular spiking input sequences with inter-spike intervals of fixed length $T$ ? Analytically integrating the coupled system (1)-(2) between two subsequent input spike times, we derive an event map

$$
\left(\begin{array}{c}
V\left(t_{m+1}\right) \\
x\left(t_{m+1}\right)
\end{array}\right)=\left(\begin{array}{c}
{\left[V\left(t_{m}\right)-V_{\mathrm{eq}}\right] e^{-T / \tau}+V_{\mathrm{eq}}+c x\left(t_{m+1}\right)} \\
{\left[x\left(t_{m}\right)(1-u)-1\right] e^{-T / \mu}+1}
\end{array}\right)
$$

from its state at input spike time $t_{m}$ to its state at the next input spike time $t_{m+1}=t_{m}+T$. Here, $V\left(t_{m}\right)$ denotes the membrane potential directly after the receiption of the spike and $x\left(t_{m}\right)$ denotes the state of the synapse directly before the transmission of the spike. The resource dynamics of this map depend only on the input interval $T$, but are independent of the potential dynamics. Thus, we directly find the fixed point of the resource dynamics defined by $x^{*}\left(t_{m+1}\right)=x^{*}\left(t_{m}\right)$. As the response map is linearly dependent on $x$ with slope $\left|(1-u) e^{-T / \mu}\right|<1$ the fixed point is globally stable. A simple analysis reveals the fixed point

$$
x^{*}[T]=\frac{1-\exp (-T / \mu)}{1-(1-u) \exp (-T / \mu)}
$$

to which the resource dynamics converges exponentially from all initial conditions. Assuming stationarity, we derive the dynamics of the membrane potential starting just after reset at $\left(V\left(t_{0}\right), x\left(t_{0}\right)\right):=\left(0, x^{*}\right)$ and consider the subthreshold membrane potential dynamics as a function of the number $n \geq 1$ of incoming spikes. Note that from this initial condition at all input spike times, we have $x\left(t_{m}\right)=x^{*}$ by construction.

Iterating the membrane potential dynamics in the event map (3) $n$ times from the initial condition $\left(V\left(t_{0}\right), x\left(t_{0}\right)\right)=\left(0, x^{*}\right)$ yields

$$
V\left(t_{n}\right)=c x^{*}[T] \sum_{m=1}^{n} e^{-(n-m) T / \tau}+V_{\text {eq }}\left(1-e^{-n T / \tau}\right) .
$$

which we prove by induction.

Induction basis: For $n=1$, (5) becomes

$$
V\left(t_{1}\right)=c x^{*}[T]+V_{\text {eq }}\left(1-e^{-T / \tau}\right)
$$

which is the first iteration of the potential dynamics in (3) for $V\left(t_{0}\right)=0, x\left(t_{0}\right)=x^{*}$ and $t_{1}-t_{0}=T$.

Induction step: Assume (5) holds for some $n \in \mathbb{N}$. Substituting (5) into (3) we obtain

$$
\begin{aligned}
V\left(t_{n+1}\right) & =\left(V\left(t_{n}\right)-V_{\text {eq }}\right) e^{-T / \tau}+V_{\text {eq }}+c x^{*}[T] \\
& =\left(c x^{*}[T] \sum_{m=1}^{n}, e^{-(n-m) T / \tau}+V_{\text {eq }}\left(1-e^{-n T / \tau}\right)-V_{\text {eq }}\right) e^{-T / \tau}+V_{\text {eq }}+c x^{*}[T] \\
& =c x^{*}[T] \sum_{m=1}^{n} e^{-(n+1-m) T / \tau}+c x^{*}[T]+V_{\text {eq }}\left(1-e^{-(n+1) T / \tau}\right) \\
& =c x^{*}[T] \sum_{m=1}^{n+1} e^{-(n+1-m) T / \tau}+V_{\text {eq }}\left(1-e^{-(n+1) T / \tau}\right)
\end{aligned}
$$

which is equation (5) with $t_{n}=n T$ replaced by $t_{n+1}=(n+1) T$. 
Evaluating the geometric sum, (5) further simplifies to

$$
V\left(t_{n}\right)=\left(1-e^{-n T / \tau}\right)\left(\frac{c x^{*}[T]}{1-e^{-T / \tau}}+V_{\text {eq }}\right)
$$

which is the membrane potential immediately after the $n$th spiking input received after potential reset at $t_{0}$.

As a consequence, the first time $t_{\tilde{n}}$ where $V\left(t_{\tilde{n}}\right) \geq 1$ defines the number of input spikes $\tilde{n}$ required for the neuron's potential to reach threshold and thus for the neuron to emit a spike. For certain combinations of system parameters and input frequencies, where $\left(\frac{c x^{*}[T]}{1-e^{-T / \tau}}+V_{\text {eq }}\right)<1$, no positive integer $\tilde{n}$ makes expression (8) larger than one. Thus, the neuron does not emit any spike and has output frequency $\lambda_{\text {out }}=0$. For all other combinations, we find

$$
\tilde{n}=\left\lceil-\frac{\tau}{T} \ln \left(1-\frac{1}{V_{\text {eq }}+\frac{c x^{*}[T]}{1-e^{-T / \tau}}}\right)\right\rceil .
$$

Here we denote by $\lceil z\rceil$ the smallest integer larger than $z$. As the input frequency of a periodic spike sequence is given by $\lambda_{\text {in }}=1 / T$ and the output period by $T_{\text {out }}=\tilde{n} T=\lambda_{\text {out }}^{-1}$, combining (9) with (4) yields

$$
\lambda_{\text {out }}=\lambda_{\text {in }} \times\left\lceil-\tau \lambda_{\text {in }} \ln \left(1-\frac{1}{V_{\text {eq }}+\frac{c_{1-(1-u) \exp \left(-1 /\left(\mu \lambda_{\text {in }}\right)\right.}}{1-\exp \left(-1 /\left(\tau \lambda_{\text {in }}\right)\right)}}\right)\right]^{-1}
$$

as the output frequency of this neuron-synapse system. This input-output relation exhibits qualitatively different shapes depending on system parameters. For instance, the neuron will spike for arbitrarily low input frequencies for one set of parameters while at others it only spikes when receiving inputs beyond a certain minimal frequency, see Fig. 2 for an illustration.

\section{Locking and limited resources suffice}

What do relations (9)-(10) tell us about the spiking response of the neuron? First, there are intervals where the output frequency is locked to the input frequency by an integer $\tilde{n}$. This locking ratio in turn changes in a jump-like manner at certain critical $\lambda_{\text {in. }}$. Thus, in the neuron's response curve, the output frequency $\lambda_{\text {out }}$ either increases proportionally to the input frequency $\lambda_{\text {in }}$ (with constant slope $1 / \tilde{n}$ ), or - at the critical frequencies $-\tilde{n}$ changes by one and the response changes qualitatively to a different proportional dependence. Interestingly, depending on the time scales in the system and the coupling constants, $\tilde{n}$ may not only decrease, but also increase by increasing of $\lambda_{\text {in }}$, thereby lowering the response frequency by increasing the input. Fig. 2 shows two qualitatively different examples. Indeed, as our prior numerical results suggested, an increase in $\tilde{n}$ and thus a downward jump in the response curve, implying its non-monotonicity, is a persistent feature in this simple neuron-synapse system.

This non-monotonic response does not rely on dynamic features of the synapse: Whereas the exact form of (10) is specific to the simple synapse-neuron system considered above, the main mechanism underlying non-monotonic input-output relations is rooted in the fact that in systems with periodic pulsed inputs and outputs locking per se occurs only between an integer number of pulses. One key observation is that a formula similar to (10) is (at least) implicitly defined across spiking neuron models, specifying the output spike frequency $\lambda_{\text {out }}$ as 

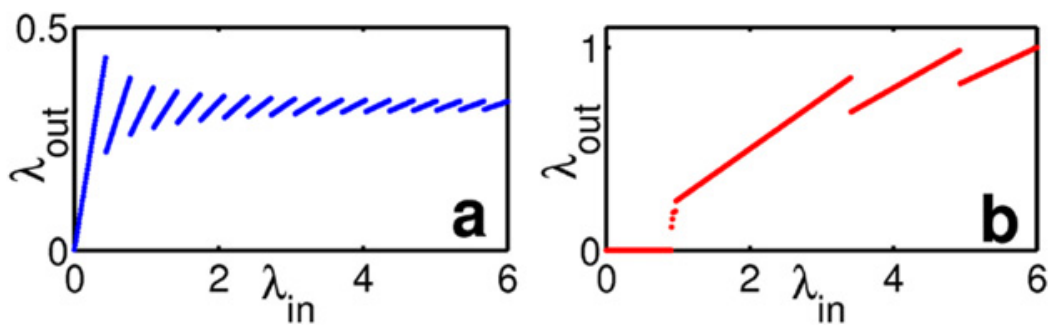

Figure 2. Non-monotonic response functions of the idealized LIF synapse-neuron system. Inputoutput response (a) for resource recovery that is much slower than the time scale of membrane potential leakage ( $\tau=1, \mu=10$ ), for system with dynamics shown in Fig. 1, (b) for both processes occurring on the same time scales ( $\tau=1, \mu=1)$. In (a) only downward jumps, in (b) upward as well as downward jumps occur. Further parameters were $u=0.2, c=0.5, V_{\text {eq }}=0.8$ for (a) and $u=0.4, c=0.8, V_{\text {eq }}=0$ for (b).

doi:10.1371/journal.pcbi.1004002.g002

a function of input frequency $\lambda_{\text {in }}$ in the regime of locking. Such an expression would be of the form

$$
\lambda_{\text {out }}=\lambda_{\text {in }} \times\left[f\left(\mathbf{p} ; \lambda_{\text {in }}\right)\right]^{-1}
$$

where $f\left(\mathbf{p} ; \lambda_{\text {in }}\right)$ is some function of all model parameters $\mathbf{p}$ and the input frequency. As for the simple LIF system above, continuously changing the input frequency $\lambda_{\text {in }}$ implies an output frequency $\lambda_{\text {out }}$ proportional to $\lambda_{\text {in }}$ within certain intervals where $f\left(\mathbf{p} ; \lambda_{\text {in }}\right)$ has real values between two integers. At frequencies $\lambda_{\text {in }}$ where $f\left(\mathbf{p} ; \lambda_{\text {in }}\right) \in \mathbb{N}$ exactly equals an integer, the response $\lambda_{\text {out }}$ jumps. Non-monotonicities emerging this way may be shielded for instance by noise, measurement errors or intrinsic obstacles to locking in complex neurons.

There are thus three conditions sufficient for this phenomenon to emerge: (i) the discrete nature of communication by pulses (such as spikes), (ii) an output pulse sequence that locks to the input sequence and (iii) any type of resource limitation in pulsetransmission.

As a consequence, it is not important where the resources are expended, whether in the presynaptic part, during intinsic processing in the postsynaptic neuron, or during action potential initiation in that neuron: in particular, non-monotonicity will also occur in neurons without dynamic synapses, as soon as any resources (e.g. ions, vesicles, neurotransmitters, calcium, or others) are needed for the spike transmission. The limited resources may thus either be expended in the presynaptic synaptic terminal leading to depressive synaptic transmissions, or may consist of the configuration of the membrane proteins which cannot change state twice within the relevant time scales. Moreover, the amount of charge required for action potential generation is limited and may be viewed as limited resources in the general theoretical sense. Finally, the notion of resources also relates to the fact that neurons exhibit a so-called refractoriness, so that immediately after the emission of a spike a neuron cannot emit a second spike due to a lack of resources.

\section{More complex neuron models}

Thus, the nonmonotonic response properties should persist in more complex neuron models and real biological neurons. We first numerically demonstrate this in two further example classes of neuronal model systems. We start with a Fitzhugh-Nagumo neuron [33] with temporally extended postsynaptic currents receiving spiking input via a static synapse. This 

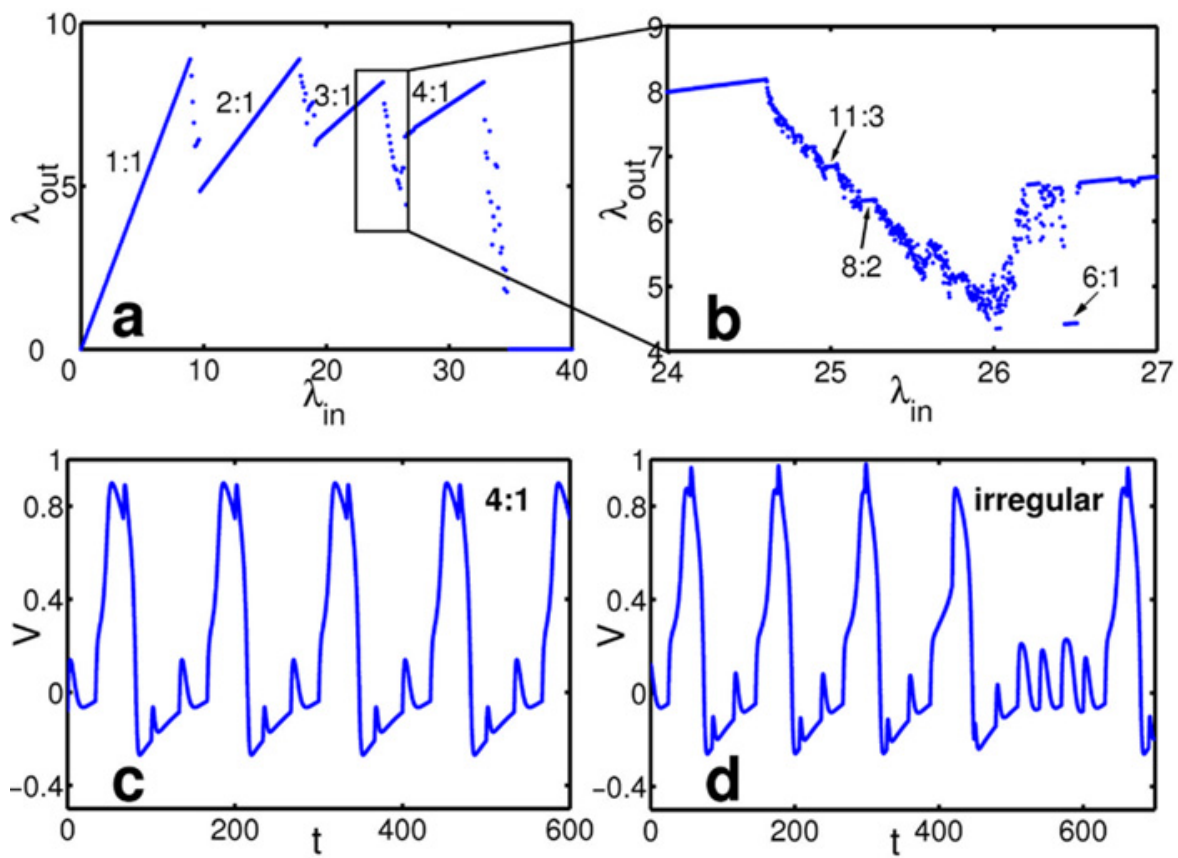

Figure 3. Non-monotonic response in a Fitzhugh-Nagumo neuron receiving periodic input via a static synapse. (a) Input-output response exhibits dominant $n: 1$-locking interrupted by broad transition regions (b), magnified from (a). Several locking ratios $n: m$ are indicated. In the transition regions, periodic, $n: m$-locked as well as nonperiodic, irregular dynamics arise. (c,d) Membrane potential dynamics (c) in the 4:1-locking region and (d) in the irregular regime. The model parameters were $a=0.139, b=2.54, c=0.5, \mu^{\prime}=125$ and $K(t)=2(\exp (-t)-\exp (-2 t))$.

doi:10.1371/journal.pcbi.1004002.g003

model is defined by the membrane potential $V(t)$ and a gating variable $W(t)$. The differential equations

$$
\begin{gathered}
\dot{V}=-V(V-1)(V-a)-W+c \sum_{m=-\infty}^{\infty} K\left(t-t_{m}\right) \\
\dot{W}=\frac{V-b W}{\mu^{\prime}}
\end{gathered}
$$

define their time evolution where $a$ is a parameter setting the equilibrium membrane potential, $b$ defines the opening rate of ion channels, $c$ is the input strength arriving via a static synapse and $\mu^{\prime}$ is related to the gating time constant. $K(t)$ is the kernel modeling the post-synaptic response to incoming spikes. The second output spike train in Fig. 1 demonstrates that the output rate can decrease with increasing input frequency in this neuron model. We studied this model systematically by recording the mean output frequency for different input frequencies in simulations. These simulations again reveal a non-monotonic input-output relation (Fig. 3a), albeit a more complicated one than for the more abstract model discussed above: As before, wherever the input is locked to the output, the response frequency necessarily increases in proportion to the input frequency. Between such bands of simple n:1-locking, we now observe extended transition regions exhibiting either more complex locking (such as, e.g., 11:3) with periodic output as well as unlocked, aperiodic output spike sequences (Fig. 3b-d). We attribute such broader transition regions to the higher-dimensional membrane potential dynamics that, together with the temporally extended spike response (and possibly numerical noise) 
includes a mechanism for the generation of spikes of different amplitudes and thus makes the system amenable of "skipping" a full spike (cf. Fig. 3c,d).

The biophysically more detailed Hodgkin-Huxley model yields qualitatively the same results, in particular non-monotonic input-output relations (cf. also third spike train in Fig. 1). As an example, Fig. 4 illustrates the input-output relation of a standard Hodgkin-Huxley neuron where we followed $[5,34]$ to describe the neuron by four dynamic variables

$$
\begin{gathered}
\dot{V}=\frac{1}{C}\left[I_{\text {ex }}+I_{0}-g_{\mathrm{Na}} m^{3} h\left(V-V_{\mathrm{Na}}\right)-g_{\mathrm{K}} n^{4}\left(V-V_{\mathrm{K}}\right)-g_{\mathrm{L}}\left(V-V_{\mathrm{L}}\right)\right] \\
\dot{m}=\left(\frac{0.1(V+40)}{1-e^{-(V+40) / 10}}\right)(1-m)-4 e^{-(V+65) / 18} m \\
\dot{n}=\left(\frac{0.01(V+55)}{1-e^{-(V+55) / 10}}\right)(1-n)-0.125 e^{-(V+65) / 80} n \\
\dot{h}=0.07 e^{-(V+65) / 20}(1-h)-\frac{1}{1+e^{-(V+35) / 10}} h,
\end{gathered}
$$

where $I_{\mathrm{ex}}$ is the input current arriving via an excitatory synapse

$$
I_{\mathrm{ex}}=\varepsilon \sum_{m} K\left(t-t_{m}\right) .
$$

In the above set of equations, $V$ is the membrane potential in $\mathrm{mV}, m, n$, and $h$ are dimensionless gating variables, $I_{\mathrm{ex}}$ is the input current in $\mu \mathrm{A} / \mathrm{cm}^{2}$ arriving via an excitatory synapse and $I_{0}$ is a constant input current in $\mu \mathrm{A} / \mathrm{cm}^{2}$. C is the membrane's capacity in $\mu \mathrm{F} / \mathrm{cm}^{2}, g_{\mathrm{Na}}, g_{\mathrm{K}}$ and $g_{\mathrm{L}}$ are the maximal specific conductances for sodium $(\mathrm{Na})$ and potassium $(\mathrm{K})$ induced currents and the leakage current through the membrane (L) in $\mathrm{mS} / \mathrm{cm}^{2} . V_{\mathrm{Na}}, V_{\mathrm{K}}$ and $V_{\mathrm{L}}$ denote the corresponding equilibrium (reversal) potentials in $\mathrm{mV} . K(\cdot)$ is the dimensionless kernel function defining the shape of the synaptic response and $\varepsilon$ specifies the strength (peak amplitude) of single inputs in $\mu \mathrm{A} / \mathrm{cm}^{2}$. Time $t$ and reception times $t_{m}$ are given in $\mathrm{ms}$.

In direct numerical simulations of this complex four-dimensional neuron model, we find input-output relations that are qualitatively similar to the responses described above. Fig. 4 shows that Hodgkin-Huxley neurons exhibit locked as well as irregular dynamics. The input-output relation increases for frequencies with locked dynamics and decreases in the transition regions with irregular dynamics, akin to those in the simpler Fitzhugh-Nagumo model. We conclude that this behaviour persists even in complex high-dimensional neuron models.

\section{Insensitivity to jitter in the input spike times}

Does the non-monotonicity of the response curve depend on the exact periodicity of the inputs? To answer this question we simulated the dynamics of the above described LIF model system with the input pulse intervals $\Delta t$ being Gamma-distributed, i.e.

$$
p(\Delta t)=\frac{\lambda^{\alpha} e^{-\lambda \Delta t} \Delta t^{\alpha-1}}{\Gamma(\alpha)}
$$

where $\lambda$ is the scale parameter and $\alpha$ is the shape parameter. The mean input interval is then $\overline{\Delta t}=\alpha / \lambda$, so that the input rate is defined by $\lambda_{\text {in }}=\lambda / \alpha$. The relative standard deviation of the input intervals is $\sigma_{\Delta t} / \overline{\Delta t}=1 / \sqrt{\alpha}$. 

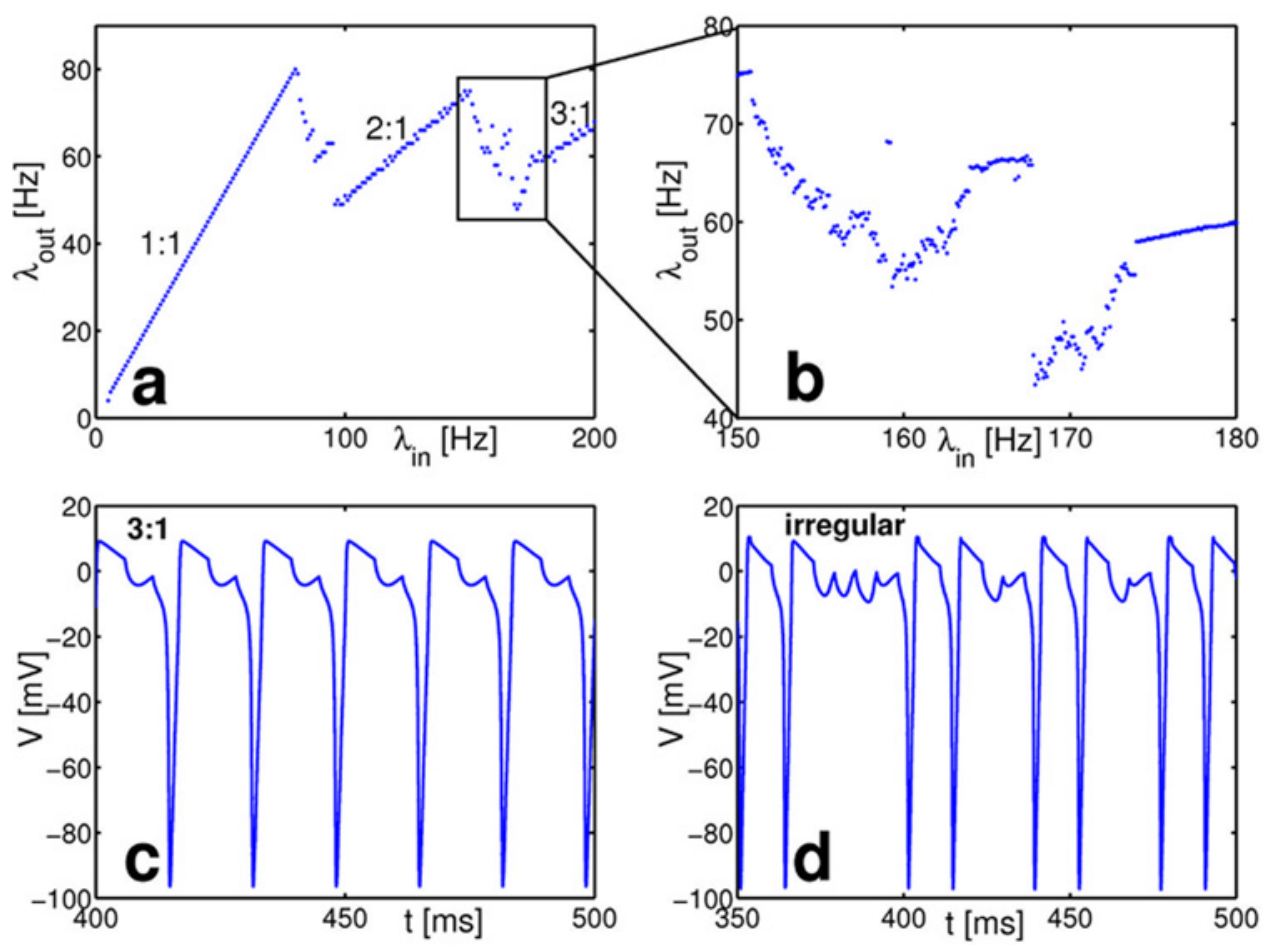

Figure 4. Non-monotonic response in a Hodgkin-Huxley neuron receiving periodic input via a static excitatory synapse. (a) In the response curve $n$ :1-locking regions are interrupted by broad transition regions (b), magnified from (a). In the transition regions nonperiodic, irregular dynamics arise. (c) and (d) show example dynamics of the membrane potential (c) in the 3:1-locking region $\left(\lambda_{\text {in }}=170 \mathrm{~Hz}\right)$ and (d) in the irregular regime $\left(\lambda_{\mathrm{in}}=140.2 \mathrm{~Hz}\right)$. Simulation parameters were $C=2, V_{\mathrm{Na}}=50, V_{\mathrm{K}}=-77, V_{\mathrm{L}}=-54.4$, $g_{\mathrm{Na}}=120, g_{\mathrm{K}}=36, g_{\mathrm{L}}=0.3, I_{0}=5$ and $\varepsilon=9$. We used an alpha-function $K(t)=e \frac{t}{\tau_{\mathrm{ex}}} \exp \left(\left(-t / \tau_{\mathrm{ex}}\right)\right)$ with time constant $\tau_{\mathrm{ex}}=1$ to model the synaptic inputs ( $e$ is the Euler constant to normalize $K(t)$ ).

doi:10.1371/journal.pcbi.1004002.g004

We again find a non-monotonic response function (see Fig. 5). Now, the infinitely fast downwards jumps (found for the deterministic input) become finite downward slopes due to the stochasticity of the input. In particular, we generally find that the non-monotonicity prevails. In the presence of any sufficiently small stochasticity the transition regions become more narrow and the frequency-decreasing jumps more pronounced the more regular the input sequences are. Based also on the theoretical finding that the main mechanism is rooted in generic resource limitation, we conclude that the phenomenon is also insensitive to fluctuations in input spike times. Only for highly irregular input the effect becomes indetectable as the dynamics are no longer locked to the input and the responses are dispersed due to the stochastic component in the input. In particular, the neuron's response function is monotonic in the limit of uncorrelated (Poisson) spike inputs. This holds for systems without any resource limitation as well as for systems with intrinsic resource limitation [39-41]. For instance, spike frequency adaptation, one specific form of resource limitation, may turn a neuron into a high pass filter with a response function that is modified compared to neurons without adaptation but still monotonic [40, 41].

We expect similarly blurred non-monotonicity for other forms of stochastic inputs modifying regular periodic input spike trains, as discussed above. For instance, spike sequence inputs with missing (skipped) spikes or sufficiently correlated spike timings may induce related nonmonotonic responses. For correlated inputs this has been, e.g., observed in [37, 38]. 

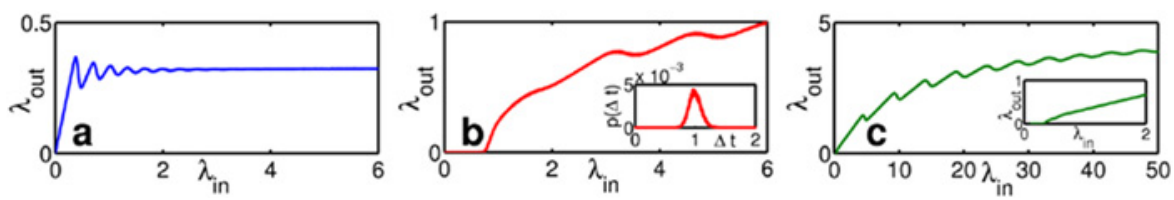

Figure 5. Non-monotonicity of response curves is robust against irregularity of the input. Panels (a)-(c) show the input-output response of the LIF model system receiving input spike sequences with Gamma-distributed inter-spike intervals. The system parameters in (a) and (b) are identical to the system parameters in Fig. 2(a),(b). (c) shows the response of the system for parameters $\tau=4, \mu=1, u=0.2, c=0.5$ and $V_{\text {eq }}=0$, where the inset demonstrates that for small input rates no output is generated. Inset of (b) shows the distribution for one fixed input rate, normalized to one. The shape parameter of the distribution was set to $\alpha=100$, so that the relative standard deviation of the input intervals is $\sigma_{\Delta t} / \overline{\Delta t}=0.1$.

doi:10.1371/journal.pcbi.1004002.g005

\section{Neurophysiological experiments: Biological neurons}

The above theory predicts generic non-monotonic responses to regular spike sequences for all neurons that exhibit at least two locking regimes. Our simulations further indicate that nonmonotonic responses persist in less perfect setups, and should thus also exist in real biological neurons. Here various types of noise and structural heterogeneities influence the neuron dynamics, specifically the exact timing of the membrane potential's response to inputs and the spike generation mechanism act as additional stochastic componentes dispersing the output spikes in time. To check if the phenomenon persists despite this dispersal due to heterogeneities, we studied neuronal responses also experimentally. For our experiments we chose neurons from the medial nucleus of the trapezoid body (MNTB) of rats. Such MNTB neurons play an essential role in the auditory pathway of rodents [35] where they support to encode and transmit features of sound stimuli. They process spike sequences of much higher regularity than those typical for basic cortical circuits [26] with input frequencies ranging from a few to several hundreds of Hertz. For this relevant frequency range we stimulated such neurons in vitro with $1 \mathrm{~ms}$ rectangular current pulses to emulate spiking inputs (see Methods section for details of slice preparation, electrophysiology and the analysis of recorded spike sequences).

Whole-cell patch-clamp recordings of MNTB neurons yielded non-monotonic response curves consistent with our theoretical predictions (Fig. 6). Panels (a) and (b) of Fig. 6 show the non-monotonic response curves for two different neurons, at three different stimulation amplitudes each. The recorded response of one (Fig. 6a) is apparently much less noisy than that of another (Fig. 6b). The overall picture is similar to the dynamics of the FHN-neurons studied above, with the exception that we did exclusively find irregular, unlocked dynamics in the transition regions, but no $n: m$-locking. We speculate that states of higher order $n: m$-locking are not sufficiently robust to be visible under such noisy conditions in inhomogeneous environments. Fig. 6(c-f) illustrates the membrane potential dynamics for the observed 1:1-, 1:2- and 1:3locking as well as for an example of unlocked, irregular response.

\section{Discussion}

The above results provide a characterization of how regular spiking inputs to neurons yield spiking output responses with universal, system-independent features. This subject was broadly neglected so far as the focus was on responses to continuous-time inputs [24, 36, 42, 43]. That neuronal dynamics can lock to the input it receives is long known and the consequence of the locked dynamics and associated changes in the neuron's response under certain conditions were observed before in specific neuronal systems [29, 30, 44-46]. Furthermore, previous studies found non-monotonic neuronal response to periodic spiking inputs $[29,30]$ and non-monotonic gain curves for neurons receiving correlated inputs via depressive synapses [37, 38]. However, 

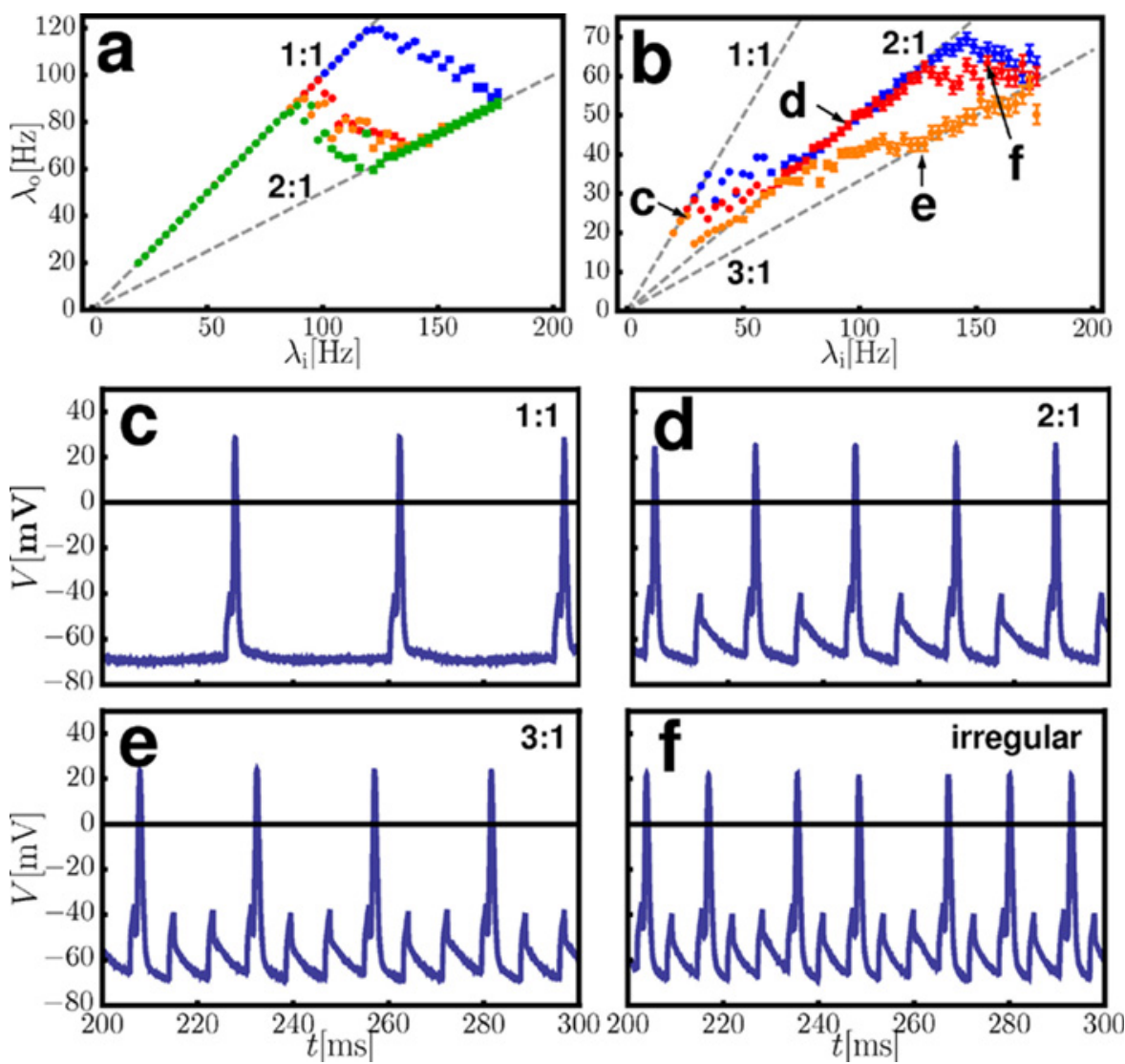

Figure 6. Non-monotonic response to regular inputs as observed in MNTB-neurons from whole-cell patch-clamp recordings. $(a, b)$ Experimentally obtained response curves of two different MNTB-neurons for different pulse currents [(a) green: $375 \mathrm{pA}$, orange, red: $425 \mathrm{pA}$, blue: $450 \mathrm{pA}$ (b) orange: $575 \mathrm{pA}$, red: $600 \mathrm{pA}$, blue: $650 \mathrm{pA}]$. The dashed lines indicate the major $n: 1$-locking states predicted —no free fit parameter. Error bars indicate the estimated error made by calculating the mean output frequency from a finite number of output spikes. (c-f) Membrane potential dynamics for different locking types: (c) 1:1-locking, (d) 2:1-locking, (e) 3:1-locking, (f) unlocked, irregular dynamics. The letters in (b) indicate the data points where these dynamics were observed.

doi:10.1371/journal.pcbi.1004002.g006

the ultimate cause for the emerging non-monotonicity as well as its generality across systems' details were not revealed in these studies. Our results now demonstrated that locking in systems that receive spiking inputs and exhibit any type of limited resource generically induces responses that are non-monotonic. We emphasize that this phenomenon relies on system-independent features and therefore is not restricted to the four systems (LIF, FHN, HH, MNTB neurons) studied here theoretically, computationally and experimentally. The above theoretical considerations demonstrate that any neuronal system exhibiting output locked to the spiking input generically exhibits non-monotonic response curves whenever resources are limited. Perfectly periodic inputs are not necessary for non-monotonic responses, but correlated inputs [37,38] suffice to excite locking in the system and thus ultimately cause non-monotonic gain curves. This finding is backed by our simulations considering jitter in the input spike times. Whereas initially revealed for systems with adaptive synapses, it is also not relevant whether resources are mainly limited in the pre-synapse or during input processing within the postsynaptic cell itself, causing, e.g., refractoriness or other effective inhibition of an action potential.

How a neuron processes regular spike sequences thus serves as a general characteristic that is complementary to its $f$ - $I$-response to continuous input currents $[10,11]$. 
Interestingly, certain neurons may exhibit a different type of non-monotonic, typically unimodal response [42, 43]. Such responses, however, are markedly distinct from the phenomenon revealed above, because potential non-monotonicities in the output rate arise as a consequence of a subthreshold resonance due to a combination of noisy and oscillating continuous-time inputs and as such are unrelated to the direct locking of discrete time spikes.

Neurons exhibiting dynamical responses similar to those illustrated above (cf. Fig. 2a) on a coarse scale display a linear transmission function with saturation threshold such that any input substantially above the threshold (about $\lambda_{\text {in }} \approx 0.3$ in our LIF example) would result in an output around that value, $\lambda_{\text {out }} \approx 0.3$, thus providing a nonlinear computational element that roughly fixates the output if the frequency of the input sequence is sufficiently high.

More generally, non-monotonic single-neuron responses will have strong functional impacts already in simple neural circuits using spike-time codes [47-49]. As an example, even a short chain, small network motifs [50] and cyclic networks of a few neurons may reliably set an operating point and classify external inputs according to their frequency. We speculate that such basic computations may be essential in neural circuits that require stabilized spike sequences and as such be of central relevance for controlling behavior. Examples for such systems operating with close-to periodic spike sequences are central pattern generators - essential for controlling locomotion - and the CA1 area of the hippocampus exhibiting oscillations with a precise and stable frequency [12-22]. In these neural networks the nonmonotonic response properties of the single neurons may well be important for the stability of the overall network dynamics. This points towards the open question how the non-monotonic relations between spiking inputs and spiking outputs of single neurons impact the computational capabilities of neural circuits in general.

\section{Methods}

The neurophysiological experiments on principal neurons of the medial nucleus of the trapezoid body (MNTB) were carried out in the following way.

\section{Slice preparation}

Brainstem slices were prepared from Wistar rats (postnatal day 8 to 10) essentially as described before in [51]: the brainstem was quickly immersed in ice-cold low $\mathrm{Ca}^{2+}$ artificial cerebral spinal fluid (aCSF) containing (in mM): $\mathrm{NaCl}$ (125), $\mathrm{KCl}(2.5), \mathrm{MgCl}_{2}$ (3), $\mathrm{CaCl}_{2}$ (0.1), glucose (25), $\mathrm{NaHCO}_{3}$ (25), $\mathrm{NaH}_{2} \mathrm{PO}_{4}$ (1.25), ascorbic acid (0.4), myo-inositol (3), Na-pyruvate (2), $\mathrm{pH}=7.3$ when bubbled with carbogen $\left(95 \% \mathrm{O}_{2}, 5 \% \mathrm{CO}_{2}\right)$. The tissue was glued onto the stage of a VT1000S vibratome (Leica, Nussloch, Germany) and $200 \mu \mathrm{m}$ thick slices were cut. Slices were transferred to an incubation chamber containing normal aCSF and maintained at $35^{\circ} \mathrm{C}$ for 30-40 $\mathrm{min}$, and thereafter kept at room temperature $\left(22-24^{\circ} \mathrm{C}\right)$ for at most 4 hours. The composition of normal aCSF was identical to low $\mathrm{Ca}^{2+}$ aCSF except that $1.0 \mathrm{mM} \mathrm{MgCl}_{2}$ and $2.0 \mathrm{mM} \mathrm{CaCl}_{2}$ were used.

\section{Electrophysiology}

Whole-cell patch-clamp recordings were obtained from MNTB-neurons using an EPC-10 amplifier. The analog signals were digitized and stored on disk using 'Pulse' software (HEKA Elektronik, Lambrecht/Pfalz, Germany). Sampling intervals and filter settings were $\leq 20 \mu$ s and 4.5 $\mathrm{kHz}$, respectively. All offline analysis was performed with 'Igor Pro' software (Wavemetrics, USA). Cells were visualized by IR-DIC microscopy through a 40x water-immersion objective (NA = 0.8) using an upright BX51WI microscope (Olympus, Germany) equipped with a 1.52x pre-magnification and a VX45 CCD camera (PCO, Germany). All experiments were carried 
out at room temperature. Patch pipettes were pulled from borosilicate glass with filament (Science Products GmbH, Hofheim, Germany) on a P-97 micropipette puller (Sutter Instrument, Novato, CA). Open tip resistance was $2-4 \mathrm{M} \Omega$ and access resistance (RS) was $\leq 20 \mathrm{M} \Omega$. Action potentials (AP) trains were elicited by injecting trains of 50 short (1 ms duration) rectangular, depolarizing current pulses at various frequencies $(20$ to $176 \mathrm{~Hz})$ and amplitudes $(250$ to $900 \mathrm{pA}$ ). The sweep interval was $200 \mathrm{~ms}$ to allow voltage-activated conductances to fully recover from inactivation. APs were measured in the current-clamp mode of the EPC-10 after adjusting the fast-capacitance cancellation while in cell-attached mode. Pipette were filled with a solution consisting of (in $\mathrm{mM}$ ): K-gluconate (100), $\mathrm{KCl}$ (60), HEPES (10), EGTA (5), Na2phosphocreatine (5), ATP-Mg (4), GTP (0.3), $\mathrm{pH}=7.3$ with $\mathrm{KOH}$.

\section{Analysis of recorded spike sequences}

We extracted the output frequency from each data trace resulting from the measurements using a self-written program. This program counted the number of output spikes $N$ generated by the MNTB neuron and registered the time $T$ between the first and the last spike. Here, we made use of the fact, that the first input spike always generated an output spike. Then, the output frequency was given by $\lambda_{\text {out }}=(N-1) / T$. As we cut off the remaining data trace, where no further output spike was measured, we made a small error $e$. We estimated the size of this error in the following way: Whenever the input is locked 1:1 to the output, there is no error as we cannot miss any output spike interval. The probability of missing one interval is proportional to the difference of input and output frequency. As we measured the neuron's output to 50 input spikes the error we make in missing one interval is $1 / 50^{\text {th }}$ of the overall measured frequency. Thus, we estimated the error to be $e=\left(\lambda_{\text {in }}-\lambda_{\text {out }}\right) / 50$.

\section{Analysis of simulated spike sequences}

As for recorded spike sequences, we counted the number $N$ of generated spikes for a time interval of length at least $\Delta t \geq \Delta t^{*}=10 s$ or 100 spikes, implying a maximum error of the output frequency of $|\Delta f| \leq(f \Delta t)^{-1}$ or $|\Delta f| f \leq 1 \%$ resp.

\section{Acknowledgments}

We thank D. Bibichkov and S. Schreiber for valuable discussions and L. Shiau for hints regarding conditions for locking. Simulation results were partly obtained using network simulation software NEST [52].

\section{Author Contributions}

Conceived and designed the experiments: HA SC SJ BU HT MT. Performed the experiments: HA SC SJ BU. Analyzed the data: HA SC SJ BU HT MT. Contributed reagents/materials/ analysis tools: HA SC SJ BU HT MT. Wrote the paper: HA HT MT. Developed the theory: HA MT.

\section{References}

1. Rieke F, Bialek W, Warland D (1999) Spikes, exploring the neural code. Cambridge, USA: MIT Press.

2. Shimokawa T, Pakdaman K, Sato S (1999) Time-scale matching in the response of a leaky integrateand-fire neuron model to periodic stimulus with additive noise. Phys. Rev. E 59: 3427. doi: 10.1103/ PhysRevE.59.3427

3. Brunel N, Chance FS, Fourcaud N, Abbott LF (2001) Effects of synaptic noise and filtering on the frequency response of spiking neurons. Phys. Rev. Lett. 86: 2186. doi: 10.1103/PhysRevLett.86.2186 PMID: 11289886 
4. Naundorf B, Geisel T, Wolf $F$ (2005) Action potential onset dynamics and the response speed of neuronal populations. J. Comput. Neurosci. 18: 297. doi: 10.1007/s10827-005-0329-8 PMID: 15830166

5. Dayan P, Abbott LF (2001) Theoretical Neuroscience. Cambridge, USA: MIT Press.

6. van Vreeswijk C, Sompolinsky H (1996) Chaos in neuronal networks with balanced excitatory and inhibitory activity. Science 274: 1724. doi: 10.1126/science.274.5293.1724 PMID: 8939866

7. Brunel N (2000) Dynamics of sparsely connected networks of excitatory and inhibitory spiking neurons. J. Comput. Neurosci. 8: 183. doi: 10.1023/A:1008925309027 PMID: 10809012

8. Roxin A, Brunel N, Hansel D (2005) Role of delays in shaping spatiotemporal dynamics of neuronal activity in large networks. Phys. Rev. Lett. 94: 238103. doi: 10.1103/PhysRevLett.94.238103 PMID: 16090506

9. Gerstner W, van Hemmen JL (1992) Universality in neural networks: The importance of the 'mean firing rate'. Biol. Cybern. 67: 195. doi: 10.1007/BF00204392 PMID: 1498186

10. Ermentrout B (1996) Type I membranes, phase resetting curves, and synchrony. Neural Comput. 8: 979. doi: 10.1162/neco.1996.8.5.979 PMID: 8697231

11. Mato G, Samengo I (2008) Type I and type II neuron models are selectively driven by differential stimulus features Neural Comput. 20: 2418.

12. Shadlen MN, Newsome WT (1994) Noise, neural codes and cortical organization. Curr. Opin. Neurobiol. 4: 569. doi: 10.1016/0959-4388(94)90059-0 PMID: 7812147

13. Softky WR (1995) Simple codes versus efficient codes. Curr. Opin. Neurobiol. 5: 239. doi: 10.1016/ 0959-4388(95)80032-8 PMID: 7620313

14. Rabinovich MI, Abarbanel HDI (1998) The role of chaos in neural systems. Neuroscience 87: 5 . doi: 10.1016/S0306-4522(98)00091-8 PMID: 9722138

15. Eurich CW, Pawelzik K, Ernst U, Cowan JD, Milton JG (1999) Dynamics of self-organized delay adaptation. Phys. Rev. Lett. 82: 1594. doi: 10.1103/PhysRevLett.82.1594

16. Diesmann M, Gewaltig MO, Aertsen A (1999) Stable propagation of synchronous spiking in cortical neural networks. Nature 402: 529. doi: 10.1038/990101 PMID: 10591212

17. Timme M, Wolf F, Geisel $\mathrm{T}$ (2002) Coexistence of regular and irregular dynamics in complex networks of pulse-coupled oscillators. Phys. Rev. Lett. 89: 258701. doi: 10.1103/PhysRevLett.89.258701 PMID: 12484926

18. Hahnloser R, Kozhevnikov A, Fee M (2002) An ultra-sparse code underlies the generation of neural sequences in a songbird. Nature 419: 224. doi: 10.1038/nature00974

19. Memmesheimer RM, Timme M (2006) Designing the dynamics of spiking neural networks. Phys. Rev. Lett. 97: 188101. doi: 10.1103/PhysRevLett.97.188101 PMID: 17155580

20. Memmesheimer RM, Timme M (2006) Designing complex networks. Physica D 224: 182. doi: 10. 1016/j.physd.2006.09.037

21. Grillner S (2006) Biological pattern generation: The cellular and computational logic of networks in motion. Neuron 52: 751. doi: 10.1016/j.neuron.2006.11.008 PMID: 17145498

22. Gong $P$, van Leeuwen $C$ (2007) Dynamically maintained spike timing sequences in networks of pulsecoupled oscillators with delays. Phys. Rev. Lett. 98: 048104. doi: 10.1103/PhysRevLett.98.048104 PMID: 17358818

23. Jahnke S, Timme M, Memmesheimer RM (2012) Giuding Synchrony Through Random Networks. Phys. Rev. X 2: 041016.

24. Jahnke S, Memmesheimer RM, Timme M (2014) Oscillation-induced signal transmission and gating in neural circuits. PLoS Comput. Biol.: accepted. doi: 10.1371/journal.pcbi.1003940

25. Jahnke S, Timme M, Memmesheimer RM (2014) A Unified Dynamic Model for Learning, Replay and Sharp-Wave/Ripples. J. Neurosci.: under review.

26. Tolnai S, Hernandez O, Englitz B, Rübsamen R, Malmierca MS (2008) The mediacal nucleus of the trapezoid body in rat: Spectral and temporal properties vary with anatomical location of the units. Eur. J. Neurosci. 27: 2587. doi: 10.1111/j.1460-9568.2008.06228.x PMID: 18547245

27. Steingrube S, Timme M, Wörgötter F, Manoonpong P (2010) Self-organized adaptation of simple neural curcuits enables complex robot behavior. Nat. Phys. 6: 224. doi: 10.1038/nphys1508

28. Luccioli S, Politi A (2010) Irregular collective behavior of heterogeneous neural networks. Phys. Rev. Lett. 105: 158104. doi: 10.1103/PhysRevLett.105.158104 PMID: 21230943

29. Perkel DH, Schulman JH, Bullock TH, Moore GP, Segundo JP (1964) Pacemaker neurons: Effects of regularly spaced synaptic input. Science 145: 61. doi: 10.1126/science.145.3627.61 PMID: 14162696

30. An Z, Yan L, Liujun C (2009) Features of hodgkin-huxley neuron response to periodic spike-train inputs. Lect. Notes Comput. Sci. 5551: 68. doi: 10.1007/978-3-642-01507-6_9 
31. Pikovsky A, Rosenblum M, Kurths J (2001) Synchronization: A universal concept in nonlinear sciences. Cambridge, USA: Cambridge University Press.

32. Tsodyks MV, Markram H (1997) The neural code between neocortical pyramidal neurons depends on neurotransmitter release probability. Proc. Natl. Acad. Sci. U. S. A. 94: 719. doi: 10.1073/pnas.94.2. 719 PMID: 9012851

33. Rocsoreanu C, Georgescu A, Giurgiteanu N (2000) The Fitzhugh-Nagumo model: Bifurcation and dynamics. Dordrecht, The Netherlands: Kluwer Academic Publishers.

34. Hodgkin A, Huxley A (1952) A quatitative description of membrane current and its application to conduction and excitation in nerve. J. Physiol. 117: 500. PMID: 12991237

35. Leao RN, Sun H, Svahn K, Berntson A, Youssoufian M, et al. (2006) Topographic organization in the auditory brainstem of juvenile mice is disrupted in congenital deafness. J. Physiol. 571: 563. doi: 10. 1113/jphysiol.2005.098780 PMID: 16373385

36. Fourcaud-Trocme N, Hansel D, van Creeswijk C, Brunel N (2003) How spike generation mechanisms determine the neuronal response to fluctuating inputs. J. Neurosci. 23: 11628. PMID: 14684865

37. Giugliano M, la Camera G, Rauch A, Lüscher H-R, Fusi S (2002) Non-monotonic current-to-rate response function in a novel Integrate-and-Fire model neuron. LNCS 2415: 141.

38. de la Rocha J, Parga N (2005) Short-term synaptic depression causes a non-monotonic response to correlated stimuli. J. Neurosci. 25: 8416. doi: 10.1523/JNEUROSCI.0631-05.2005 PMID: 16162924

39. Liu Y-H, Wang X-J (2001) Spike-frequency adaptation of a generalized Leaky Integrate-and-Fire model neuron. J. Comput. Neurosci. 10: 25. doi: 10.1023/A:1008916026143 PMID: 11316338

40. Benda J, Herz AVM (2003) A universal model for spike-frequency adaptation. Neural Comput. 15: 2523. doi: 10.1162/089976603322385063 PMID: 14577853

41. Rauch A, la Camera G, Lüscher H-R, Senn W, Fusi S (2003) Neocortical pyramidal cells respond as Integrate-and-Fire neurons to in vivo-like input currents. J. Neurophysiol. 90: 1598. doi: 10.1152/jn. 00293.2003 PMID: 12750422

42. Schreiber S, Erchova I, Heinemann U, Herz AVM (2004) Subthreshold resonance explains the frequency-dependent integration of periodic as well as random stimuli in the entorhinal cortex. J. Neurophysiol. 92: 408. doi: 10.1152/jn.01116.2003 PMID: 15014100

43. Engel TA, Schimansky-Geier L, Herz AVM, Schreiber S (2008) Subthreshold membrane-potential resonances shape spike-train patterns in the entorhinal cortex. J. Neurophysiol. 100: 1576. doi: 10.1152/jn. 01282.2007 PMID: 18450582

44. Guttman R, Feldman L, Jakobsson E (1980) Frequency entrainment of squid axon membrane. J. Membrane Biol. 56: 9. doi: 10.1007/BF01869347

45. Longtin A, Bulsara A, Pierson D, Moss F (1994) Bistability and the dynamics of periodically forced sensory neurons. Biol. Cybern. 70: 569. doi: 10.1007/BF00198810 PMID: 8068770

46. Engelbrecht JR, Mirollo R (2009) Dynamical phase transitions in periodically driven model neurons. Phys. Rev. E 79: 021904.

47. Singer W (1999) Time as a coding space? Curr. Opin. Neurobiol. 9: 189. doi: 10.1016/S0959-4388(99) 80026-9

48. VanRullen R, Guyonneau R, Thorpe SJ (2005) Spike times make sense. Trends Neurosci. 28: 1. doi: 10.1016/j.tins.2004.10.010 PMID: 15626490

49. Masquelier T, Guyonneau R, Thorpe SJ (2008) Spike timing dependent plasticity finds the start of repeating patterns in continuous spike trains. PLoS One 3: e1377. doi: 10.1371/journal.pone.0001377 PMID: 18167538

50. Sporns O, Kötter R, (2004) Motifs in brain networks PLoS Biol. 2: e369. doi: 10.1371/journal.pbio. 0020369

51. Taschenberger H, von Gersdorff $\mathrm{H}$ (2000) Fine-tuning an auditory synapse for speed and fidelity: Developmental changes in presynaptic waveform, EPSC kinetics, and synaptic plasticity. J. Neurosci. 20: 9162. PMID: 11124994

52. Gewaltig M.O., Diesmann M. (2007) NEST (NEural Simulation Tool). Scholarpedia 2: 1430. doi: 10. 4249/scholarpedia.1430 